\title{
ORIGINAL ARTICLE \\ Outcomes after acute traumatic spinal cord injury in Botswana: from admission to discharge
}

\author{
I Löfvenmark ${ }^{1,2}$, M Hasselberg ${ }^{3}$, L Nilsson Wikmar ${ }^{1}, \mathrm{C}$ Hultling ${ }^{1,4}$ and C Norrbrink ${ }^{1}$
}

Study design: Prospective follow-up study.

Objectives: To increase the knowledge regarding clinical and functional outcomes after traumatic spinal cord injuries (TSCI) in a resource-constrained setting. A special focus was placed on secondary complications such as pressure ulcers.

Setting: The national referral hospital in Gaborone, Botswana.

Methods: The study included all patients admitted with an acute TSCI during a 2-year period $(n=39)$. Data collection was conducted at the time of discharge, and clinical characteristics, length of stay and pressure ulcers were analysed using descriptive and inferential statistics.

Results: Completeness of injury and presence of pressure ulcers were the factors found to significantly prolong hospitalization, which was 5 months (median). One patient died before discharge and one was discharged to rehabilitation in South Africa; all other patients were living with close or distant family members after discharge. Patients were supplied with electrical or manual active wheelchairs. Self-catheterization or suprapubic catheters were the main methods for bladder management, and ano-rectal stimulation to manage the bowel. Pressure ulcers, urinary tract infections and pain were the most frequent complications during in-patient care.

Conclusions: Rehabilitation of patients with TSCl in Botswana has been going through big changes, and new rehabilitation objectives, such as techniques used for the management of bladder and bowel dysfunctions and the provision of technical aids, have been implemented, which likely can contribute to an overall improvement in the outcomes. However, basic care at the general wards is still lagging behind, causing high rates of pressure ulcers that significantly extend hospitalization periods.

Spinal Cord (2017) 55, 208-212; doi:10.1038/sc.2016.122; published online 16 August 2016

\section{INTRODUCTION}

A spinal cord injury (SCI) is a life-changing event regardless of where you live. Having access to services such as specialized acute management and comprehensive rehabilitation impacts functional outcomes, quality of life and the rates of secondary complications ${ }^{1}$ and survival. ${ }^{2}$ Despite great improvements in the management of acute traumatic SCI (TSCI), resource-constrained settings have not benefitted from this progress to the same extent as have countries with vast resources.

In-hospital mortality rates after acute TSCI are higher in resource-constrained settings, ${ }^{1,3}$ mainly due to pressure ulcers (PU) and urinary tract infections (UTI). ${ }^{1,4}$ Respiratory complications, ${ }^{5,6}$ delays in admissions ${ }^{7}$ and multiple hospital presentations also contribute to the high mortality. ${ }^{1,7}$ Mortality rates in sub-Saharan Africa have been reported to be between 17 and 29\%, ,7,8 compared with close to zero in settings with more resources. ${ }^{2}$

Prevention of PU is a major challenge especially in areas where pressure relief mattresses are lacking, knowledge among staff is limited, and hospitals are understaffed, which challenges frequent turnings. ${ }^{5,9} \mathrm{PU}$ and UTI are reported to range between $35-57 \%$ and $25-62 \%$, respectively. ${ }^{5,6,8,9} \mathrm{PU}$ also often lead to prolonged hospitalization, ${ }^{6,9}$ which is reported to be $3-5$ months in resource-constrained settings ${ }^{5,6,9}$ and 2-3 months in high-income countries. ${ }^{10,11}$ Pain has been reported in large surveys as the major secondary complication to SCI. ${ }^{4,12}$
The World Health Organization (WHO) defines handicap as 'a disadvantage for a given individual resulting from an impairment or a disability that limits or prevents the fulfilment of a role that is normal for that individual'- that is, how it reflects on the cultural, social, economic and environmental factors. ${ }^{13}$ Thus, the same disability leads to different levels of handicap depending on where the person lives. Wheelchairs can be enormously challenging to acquire in many parts of the world and the ones that are available are rarely constructed for the inaccessible environment. ${ }^{1,4}$ Clean intermittent catheterization (that is, self-catheterization) decreases complication rates compared with indwelling catheters and is recommended for the management of neurogenic bladder dysfunction. ${ }^{14}$ However, indwelling catheters are used to a large extent in resource-constrained settings because of a lack of catheters and staff to assist with catheterization, and a resistance among patients to self-catheterize. ${ }^{1,4}$ Faecal incontinence is also common in these areas where structured bowel programmes are obstructed by the use of squat toilets or where people simply go out to the fields, ${ }^{1}$ even though, for example, inexpensive accessible pit-latrines or commode chairs might be constructed.

Botswana's development, from one of the poorest African countries to a middle-income country, has been rapid, and a relatively well-developed health-care system is in place today. There is a lack of information from Botswana, as well as from most sub-Saharan African countries, with regard to the management of TSCI. The main

${ }^{1}$ Division of Physiotherapy, Department of Neurobiology, Care Sciences and Society, Karolinska Institutet, Stockholm, Sweden; ${ }^{2}$ Spinalis/Rehab Station Stockholm, Stockholm, Sweden; ${ }^{3}$ Department of Public Health Sciences, Karolinska Institutet, Stockholm, Sweden and ${ }^{4}$ Division of Neurodegeneration, Department of Neurobiology, Care Sciences and Society, Karolinska Institutet, Stockholm, Sweden

Correspondence: I Löfvenmark, Spinalis, Rehab Station Stockholm, Frösundaviks allé 13, Solna, Stockholm 169 89, Sweden.

E-mail: inka@spinalis.se

Received 1 March 2016; revised 10 June 2016; accepted 1 July 2016; published online 16 August 2016 
aim of this study was to increase the knowledge regarding clinical and functional outcomes after TSCI in Botswana. A special focus was placed on secondary complications such as PU.

\section{MATERIALS AND METHODS}

\section{Setting and population}

This prospective follow-up study was conducted at the Spinalis SCI-rehabilitation centre established in 2010 at the Princess Marina Hospital $(\mathrm{PMH})$ in Botswana. This is the national referral hospital for TSCI to where all patients with TSCI should be transferred. Acute care, before patients being surgically stabilized and medically stable, is managed at the intensive care unit and the orthopaedic wards. Thereafter, the patients are transferred to the SCI-rehabilitation centre. The SCI centre is an 8- to 12-bed unit depending on the severity of injuries among the patients and is staffed with local professionals: 2 medical doctors, 10-12 nurses, 2 physiotherapists, 2 occupational therapists, 1 rehabilitation coach and 6 health-care auxiliaries.

All patients who were admitted with an acute TSCI during a 2-year period, from 1 February 2011 to 31 January $2013(n=49)$, and survived the acute phase and were transferred to the rehabilitation centre $(n=39)$ were included and followed through the discharge process. The admission phase of this cohort has been described in a previously published paper, ${ }^{15}$ which showed an estimated annual incidence rate of 13 persons per million, with road traffic crashes being the cause in $68 \%$, of which the majority were single accidents. Mortality before transfer to the SCI-rehabilitation centre was $20 \%$. The paper also reported a $59 \%$ tetraplegia rate and revealed that the time from injury to

Table 1 Demographic and clinical characteristics at admission and discharge

\begin{tabular}{|c|c|c|c|c|}
\hline & \multicolumn{2}{|c|}{ Admission, $\mathrm{n}=49$} & \multicolumn{2}{|c|}{ Discharge, $\mathrm{n}=39$} \\
\hline & $\mathrm{n}$ & $\%$ & $n$ & $\%$ \\
\hline \multicolumn{5}{|l|}{ Gender $^{a}$} \\
\hline Male & 36 & 73 & 27 & 69 \\
\hline Female & 13 & 27 & 12 & 31 \\
\hline \multicolumn{5}{|l|}{ Marital status ${ }^{b}$} \\
\hline Married/cohabit & 11 & 28 & 12 & 31 \\
\hline Single & 26 & 67 & 25 & 64 \\
\hline Children $(<10$ years of age) & 2 & 5 & 2 & 5 \\
\hline \multicolumn{5}{|l|}{ Aetiology } \\
\hline Traffic related & 33 & 68 & 24 & 62 \\
\hline Assault & 8 & 16 & 8 & 20 \\
\hline Falls & 5 & 10 & 4 & 10 \\
\hline Sports or struck by objects & 3 & 6 & 3 & 8 \\
\hline \multicolumn{5}{|l|}{ Type of injury } \\
\hline C1-8 AIS A,B,C & 15 & 31 & 10 & 26 \\
\hline T1-S5 AIS A,B,C & 18 & 37 & 17 & 44 \\
\hline All AIS D & 8 & 16 & 11 & 28 \\
\hline Tetraplegia unclear AIS & 7 & 14 & - & - \\
\hline Paraplegia unclear AIS & 1 & 2 & $1^{\mathrm{c}}$ & 2 \\
\hline Deceased & $10^{d}$ & 20 & $1^{\mathrm{e}}$ & 2 \\
\hline
\end{tabular}

Abbreviations: AIS, American spinal injury association Impairment Scale; C, cervical; S, sacral; $\mathrm{T}$, thoracic.

aNumbers differ from the previously published study ${ }^{15}$ due to an error in the database regarding gender; one male was indicated as female. When this was corrected no significant gender differences were found $(0.09)$.

differences were found (0.09).

Admission data exclude deceased patients $(n=10)$.

Included in the T1-S5 AIS A, B and C group for analysis as some function was preserved in

the lower extremities, bladder and bowel control, but the patient was not ambulating.

${ }^{d}$ Deceased during acute phase.

eIncluded in all analysis. stabilizing surgery was long (median $=12$ days). The demographic and clinical characteristics of the patients are shown in Table 1.

\section{Data collection and analysis}

The severity of injury was assessed according to the International Standards for the Neurological Classification of Spinal Cord Injury. ${ }^{16}$ The International SCI data sets ${ }^{17}$ were used, including the core data set, and those for lower urinary tract function, bowel function and pain. Functional Independence Measure (FIM) was used for functional performance. Data collection was conducted at discharge assessments and complemented with socio-demographic information, complications and mode of mobility. The injury severity categories used were as follows: C1-8 American spinal injury association Impairment Scale (AIS) A, B and C; T1-S5 AIS A, B and C; and all AIS D. ${ }^{18}$ The length of stay (LOS) included total admission at the $\mathrm{PMH}$, including the intensive care unit, the orthopaedic ward and the surgical ward, and the duration at the rehabilitation centre.

Categorical variables are presented as absolute numbers and proportions, and continuous variables are presented as mean, s.d., median and range or interquartile range (IQR). ${ }^{18}$ Differences between groups were analysed using the non-parametric Mann-Whitney $U$-test for continuous variables and the Kruskal-Wallis test for comparisons with more than two groups. Fisher's exact test was used for categorical variables with small samples, and Spearman for correlations between LOS and age because of small numbers. The $P$-value for statistical significance was set to $P<0.05$. SPSS statistics version 21 (IBM, New York, NY, USA) was used for statistical analysis.

Ethical approval has been obtained from the Ministry of Health in Botswana (PPME: 13/18/1 Vol VIII (92)) and at Princess Marina Hospital (PMH 5/79 (27a)). Informed consent forms were obtained from all participants.

\section{RESULTS}

In total, 39 patients were included in the study. The median age at injury was 31 years (range 4-62, IQR 23-40). The majority of patients were male and injuries were mainly traffic related. Demographic and clinical characteristics of the patients are presented in Table 1 .

\section{LOS and place of discharge}

The median LOS at the PMH was 20 weeks (see Table 2). For patients with tetraplegia $(n=20)$ the LOS was 13 weeks (IQR 9-22) and for those with paraplegia $(n=19)$ the LOS was 22 weeks (IQR 13-29) $(P=0.140)$. Completeness of injury $(P=0.002)$ and the presence of PU $(P=0.001)$ significantly extended LOS (see Table 2$)$, whereas gender, pain (see Table 2), age and marital status (results not shown in the table) did not.

One ventilator-dependent patient with a complete C3 injury died before the planned discharge, $\sim 6$ months post injury (unclear reason). All remaining patients were, after being discharged, living with close or extended families, except for one patient who went for rehabilitation to South Africa. The house modifications that had been done before discharge included a few ramps that had been constructed by the families.

\section{Outcomes}

Neurological recovery of at least one AIS-grade improvement was seen in six patients (16\%). FIM motor scores at discharge assessment are presented in Table 3. Patients requiring mobility aids (Table 3) were supplied with electrical or custom-made active manual wheelchairs (through Motor Vehicle Accident Fund or government funds) according to recommendations given by the physio- and occupational therapists. For bladder management, self-catheterization or suprapubic catheters (depending on the level of injury) were the methods used by the majority of patients with neurogenic bladder dysfunction, and digital ano-rectal stimulation by those with bowel dysfunction (Table 3). No bladder leakage or bowel incontinence was reported by 26 versus 20 patients (missing data $=9$ versus 10). Bowel 
Table 2 Length of stay at Princess Marina Hospital by type of injury, gender, associated injuries and complications

\begin{tabular}{|c|c|c|c|c|c|c|}
\hline & \multicolumn{6}{|c|}{ Weeks } \\
\hline & $\mathrm{n}$ & Mean & s.d. & Median & $I Q R$ & $\mathrm{P}$-value \\
\hline Type of injury at discharge & & & & & & $0.002^{\mathrm{b}}$ \\
\hline C1-8 AIS A,B,C & 10 & 23 & 10 & 22 & $18-32$ & \\
\hline T1-S5 AIS A,B,C & 18 & 23 & 11 & 24 & $14-30$ & \\
\hline All AIS D & 11 & 10 & 4 & 10 & $9-12$ & \\
\hline Total & 39 & 19 & 11 & 20 & 10-27 & \\
\hline Gender & & & & & & $0.503^{c}$ \\
\hline Male & 27 & 20 & 11 & 21 & $9-29$ & \\
\hline Female & 12 & 17 & 9 & 16 & $11-26$ & \\
\hline Associated injury & & & & & & $0.283^{c}$ \\
\hline Yes & 13 & 22 & 10 & 22 & $14-29$ & \\
\hline No & 26 & 18 & 11 & 14 & $9-26$ & \\
\hline Pressure ulcers & & & & & & $0.001^{c}$ \\
\hline Yes & 16 & 26 & 9 & 26 & $19-30$ & \\
\hline No & 23 & 15 & 10 & 12 & $9-22$ & \\
\hline Pain & & & & & & $0.559^{c}$ \\
\hline Yes & 27 & 19 & 10 & 21 & $10-27$ & \\
\hline No & 9 & 22 & 14 & 17 & $11-34$ & \\
\hline Missing data & 3 & 11 & 9 & 9 & - & \\
\hline
\end{tabular}

Abbreviations: AIS, American spinal injury association Impairment Scale; C, cervical; $I Q R$, interquartile range; $S$, sacral; $T$, thoracic.

IQR, interquartile range; $\mathrm{S}$, sacral; $\mathrm{T}$,
Bold indicates significant differences.

aFor analysis of LOS, the rank order was based on days (based on the assumption of a 30-day month), while the presented values are converted to weeks.

${ }^{b}$ Kruskal-Wallis test.

cMann-Whitney U-test.

management was performed in bed by three patients, whereas the rest used a toilet or commode chairs. FIM scores for bladder and bowel management show that 28 and 25 patients, respectively, were independent or modified independent.

\section{Consequences and complications}

In all, 7 out of the 21 patients with spastic paralysis considered the spasticity to be problematic (missing data $=7$ ). The most prevalent complications were PU $(n=16)$, UTI $(n=11)$ and pain $(n=27)$. Additionally, a few cases of heterotopic ossification, respiratory complications and deep vein thrombosis were seen (Table 4). At least 1 wound was observed in 19 patients; of them, 16 patients developed PU, of whom 2 underwent plastic surgery, 3 had surgical wound complications and 2 patients had burn injuries. Five patients had developed PU before admission to PMH (sacral, heel or elbow), 8 patients developed $\mathrm{PU}$ at other wards at $\mathrm{PMH}$ before transferral to the rehabilitation centre (sacral, heels or occiput), and 3 developed PU during rehabilitation. The PU developed at the SCI-rehabilitation centre came from the backrest of the wheelchair or from lying in bed (sacral). The severity of injury did significantly affect the rate of PU $(P=0.014)$; however, the neurological level of lesion did not $(P=1.0)$ (see Table 5). Out of the 16 patients with PU, 13 were classified as AIS A. The motor FIM score also differed significantly between the groups with a median of 69 (IQR 54-83) for the group who had developed PU and 84.5 (IQR 70-91) for those who did not $(P=0.026)$.

\section{DISCUSSION}

The main findings of this study show that bladder and bowel management methods, the provision of appropriate technical aids
Table 3 Functional outcomes at discharge after TSCI $(n=39)$

FIM motor score ${ }^{\mathrm{a}}$ ( $n$; missing data)

C1-8 AIS A,B,C $(8 ; 2)$

Mean (s.d.) 43

C1-8 AIS A,B,C $(8 ; 2)$

Median (IQR) 43

Mean (s.d.) 75

-S5 AIS A,B,C (16; 2)

Median (IQR) 82

Mean (s.d.) 88.5

All AIS D $(8 ; 3)$

Median (IQR) 91

All AIS D $(8 ; 3)$

$\begin{array}{rr}n & \% \\ 23 & 59 \\ 10 & 26 \\ 6 & 15\end{array}$

Mobility

Manual or electrical wheelchair

Ambulatory

Partly ambulatory and wheelchair-users

6

Bladder management method

Normal function

Indwelling catheter

Suprapubic catheter

Clean intermittent catheterization ${ }^{\mathrm{b}}$

Condom catheter

Missing data

$\begin{array}{cc}10 & 26 \\ 6 & 15 \\ 7 & 18 \\ 10 & 26 \\ 1 & 3 \\ 5 & 12\end{array}$

Bowel management method

Normal function

Digital stimulation

17

Colostomy

Otherc

Missing data
43
43
75
82
8.5
91
$\%$
59
26
15

26
15
18
26
3
12

26

2
Abbreviations: AIS, American spinal injury association Impairment Scale; C, cervical; FIM, functional independence measure; IQR, interquartile range; S, sacral; T, thoracic; $\mathrm{TSCl}$, traumatic spinal cord injury.

aFIM motor score (\#1-13), minimum-maximum score 13-91.

${ }^{b}$ Clean intermittent catheterization was performed by the attendant for one child.

'Digital evacuation, enema and straining were other bowel management methods.

and mortality during rehabilitation in Botswana have become more like the situation in many high-income countries, whereas LOS and the presence of PU remain similar to other resourceconstrained settings.

LOS in our study was comparable to that in other resourceconstrained settings (3-5 months), ${ }^{5,6}$ with longer stays for patients with complete injuries, which is in line with previous studies. ${ }^{10,19}$ Surprisingly, patients with paraplegia had longer LOS, probably due to the fact that all but one of the ambulating patients (AIS D) had a tetraplegia. PU resulted in a prolonged LOS, which also corresponds with the results of previous studies, ${ }^{6,9,20}$ whereas pain did not. However, comparing LOS is challenged by the fact that care at $\mathrm{PMH}$ incorporates acute management (at the intensive care unit and orthopaedic wards), rehabilitation, and for most patients a 2- to 4week home leave before discharge. These home leaves were initiated because of the vast distances to the sites of discharge: up to 900 kilometres. There might also be other indicators delaying the time to discharge, such as providing transport, lack of nurses to escort the patient home, administrative issues and the irregular presence of a social worker. All patients, but one, were living with close or extended families after discharge, often at different settings compared with pre-injury. Botswana does not have nursing homes or such institutions that can lead to occasional discharges to families reluctant to accept the injured person home because of the extra burden it creates. The strains on the caregiver and families are considerable, and patients who deliberately resist wound healing or improvement in order to extend their stay at the hospital have been reported, ${ }^{4}$ but were not identified in our study. 
Table 4 Complications during in-patient care by type of injury

\begin{tabular}{|c|c|c|c|c|}
\hline & $\begin{array}{c}C 1-8 \\
\text { AIS A,B,C }(\mathrm{n}=10)\end{array}$ & $\begin{array}{c}\text { T1-S5 } \\
\text { AIS A,B,C ( } \mathrm{n}=18)\end{array}$ & $\begin{array}{c}A l l \\
A / S D(\mathrm{n}=11)\end{array}$ & Total, $\mathrm{n}=39(\%)$ \\
\hline Complications (excluding pain)a & 7 & 14 & 2 & $23(59)$ \\
\hline Pressure ulcers & 5 & 10 & 1 & $16(41)$ \\
\hline Surgical wound complications & 0 & 3 & 0 & $3(8)$ \\
\hline Burn wounds & 1 & 1 & 0 & $2(5)$ \\
\hline Missing data & 1 & 4 & 2 & $7(18)$ \\
\hline Pain & 7 & 12 & 8 & $27(69)$ \\
\hline Of which shoulder pain & 5 & 0 & 4 & $9(33)$ \\
\hline Missing data & 1 & 1 & 1 & $3(8)$ \\
\hline Heterotopic ossification/myositis & 0 & 3 & 0 & $3(8)$ \\
\hline Respiratory & 3 & 0 & 0 & $3(8)$ \\
\hline
\end{tabular}

Abbreviations: AIS, American spinal injury association Impairment Scale; C, cervical; T, thoracic; S, sacral.

${ }^{a}$ Any complication including pain was present in 34 out of 39 patients.

bMultiple complications exclude pain. Nine patients had both wound/s and urinary tract infection.

Table 5 Development of pressure ulcers during in-patient care by type of injury

\begin{tabular}{|c|c|c|c|c|c|}
\hline \multirow[t]{2}{*}{ Type of injury } & \multicolumn{2}{|c|}{$\begin{array}{l}\text { Pressure ulcer/s, } \\
\qquad \mathrm{n}=16\end{array}$} & \multicolumn{2}{|c|}{$\begin{array}{l}\text { No pressure ulcer, } \\
\qquad \mathrm{n}=23\end{array}$} & \multirow[t]{2}{*}{$P$-value ${ }^{a}$} \\
\hline & $n$ & $\%$ & $\mathrm{n}$ & $\%$ & \\
\hline C1-8 AIS A,B,C & 5 & (31) & 5 & (22) & 1.0 \\
\hline T1-S5 AIS A,B,C & 10 & (63) & 8 & (35) & 0.014 \\
\hline All AIS D & 1 & $(6)$ & 10 & (43) & \\
\hline
\end{tabular}

Abbreviations: AIS, American spinal injury association Impairment Scale; C, cervical; S, sacral; $\mathrm{T}$, thoracic.

Bold indicates significant differences.

aFisher's exact test.

Respiratory complications such as pneumonia and influenza together with cardiovascular disease are the main causes of death after TSCI in high-income countries. ${ }^{3}$ In resource-constrained settings, PU and UTI cause high mortality rates. ${ }^{1,3}$ The one patient who died at the rehabilitation centre was the first ventilator-dependent patient discharged from the intensive care unit and was prepared to be discharged home with a portable ventilator. Thus, mortality due to infections or wounds was non-existent, even though mortality before referral to the SCI-rehabilitation centre at PMH was high. ${ }^{15}$

Physicians with long-term experience in TSCI management in Botswana have described the challenging situation that has lasted for a long time and up until the SCI-rehabilitation centre was initiated. ${ }^{8}$ Appropriate wheelchairs and cushions were rare, indwelling catheters were the common method for bladder management, and patients with tetraplegia were often viewed as lacking rehabilitation potential, resulting in discharge from the general wards after completed caregiver training. One multi-centre study showed that the rehabilitation objectives of a unit and staff density were two factors that affected the outcomes. ${ }^{21}$ These are two factors that have been upgraded in TSCI management in Botswana during the last years that probably have had a positive impact on the outcomes together with an increase in knowledge among SCI staff. Patients were supplied with appropriate wheelchairs and cushions (when delivery was delayed, temporary wheelchairs and cushions were provided). The use of self-catheterization and suprapubic catheters has been successfully introduced and was practised at nearly comparable rates with settings with more resources (38 and 21\% respectively in Sweden) ${ }^{19}$ and might have contributed to the relatively low rate of UTI $(28 \%) .{ }^{14}$ Single-time use of catheters was practised during in-patient care; however, in the home environment non-coated catheters were recommended for multiple uses due to the challenges in maintaining a continuous supply. Six patients were discharged with an indwelling catheter because of their reluctance both to perform self-catheterization and to have a suprapubic catheter or because catheters were lacking. Bowel management was improved from being done in bed or using a colostomy to practising ano-rectal stimulation on the toilet, commode or pit-latrine.

$\mathrm{PU}$ are reported at a higher rate from resource-constrained settings (35-57\%) compared with settings with more resources $(24 \%), 5,9,11$ even though higher rates are reported from resource-rich settings as well, ${ }^{22}$ and are associated with completeness of injury. ${ }^{8,22}$ Zakrasek et $a l .{ }^{20}$ identified preventive measures such as education, involvement and education of family, increased nurse-to-patient ratio, optimized nutrition, maintaining good skin hygiene, having pressure relief surfaces and finally having 'turning teams' or other ways of regular pressure relief. ${ }^{4}$ In our study, $41 \%$ were treated for PU, with the majority having developed PU before being transferred to the SCI-rehabilitation centre. As reported in a previous study, the median waiting time for surgery was 12 days, which increases the risk for $\mathrm{PU}$ as manpower and knowledge are required to perform log-rolling for patients with spinal instabilities. At the SCI centre, wounds were healed with pressure relief routines, wound care and dietician consultations; however, these measures were generally lacking at the general wards. All wounds, except one surgical wound, one burn and one PU, were healed at discharge. Having hospital 'turning teams' has been discussed and advised by local physicians; however, installing staff positions has not been successful.

\section{Strength and limitations}

The strength of this study is the national approach, with $\mathrm{PMH}$ being the national referral hospital for patients with acute TSCI. 
This new structure was implemented from the government simultaneously with the establishment of the Spinalis SCIrehabilitation centre, and data collection started $\sim 1$ year later. However, the centre moved to its own premises from being a part of the orthopaedics ward within the study period, especially complicating the analysis on LOS. It was not feasible to separate the patients' acute care phase from the rehabilitation phase, which is why the total LOS at PMH is reported.

An additional limitation that needs to be addressed includes the use of FIM, which has been debated and is considered to be too indiscrete to capture progress in the SCI population. However, it was in use at the centre and was therefore included in the study, which might lead to an occasional lack of detected changes. Changing the protocol was discussed, but was not feasible at the time. Additionally, no admission FIM had been conducted, due to which the functional improvement could not be evaluated. Furthermore, pain was often not specified and could not be classified-that is, determination of neuropathic or nociceptive pain. Reasons for this are several, including insufficient knowledge due to the initially rotating medical officers and deficiencies of filling out protocols and conducting assessments. However, the study still highlights the high frequency of pain that needs to be addressed during rehabilitation. Finally, the statistical analysis should be interpreted with caution because of the small sample of patients included. Despite the above-mentioned limitations, this study provides new knowledge about the management of patients with acute TSCI from sub-Saharan Africa. Future research with longer follow-up periods in order to facilitate further development of specialized care is needed.

In conclusion, the rehabilitation of patients with TSCI in Botswana has, to some extent, become more like the situation in environments with more resources, such as successfully implemented new rehabilitation objectives-for example, techniques used for the management of bladder and bowel dysfunctions and the provision of technical aids, which can contribute to an overall improved outcome and decreased secondary complications. Additionally, the mortality rate due to secondary complications was low. On the other hand, the basic care at the general wards corresponds more with what is expected in resource-constrained settings, contributing to high rates of PU that significantly extend hospitalization periods.

\section{DATA ARCHIVING}

There were no data to deposit.

\section{CONFLICT OF INTEREST}

The authors declare no conflict of interest.

\section{ACKNOWLEDGEMENTS}

We would like to thank all included patients and the staff at the Spinalis SCIrehabilitation centre in Botswana. We also thank Neuro Sweden and Spinalis Foundation for financial support and Dirk Casto for editing the manuscript.

1 Burns AS, O'Connell C. The challenge of spinal cord injury care in the developing world. J Spinal Cord Med 2012; 35: 3-8.

2 Divanoglou A, Westgren N, Seiger A, Hulting C, Levi R. Late mortality during the first year after acute traumatic spinal cord injury: a prospective, population-based study. J Spinal Cord Med 2010; 33: 117-127.

3 WHO. International Perspectives on Spinal Cord Injury, Summary. WHO: Geneva, Switzerland, 2013.

4 Levy LF, Makarawo S, Madzivire D, Bhebhe E, Verbeek N, Parry O. Problems, struggles and some success with spinal cord injury in Zimbabwe. Spinal Cord 1998; 36: 213-218.

5 Obalum DC, Giwa So, Adekoya-Cole TO, Enweluzo GO. Profile of spinal injuries in Lagos, Nigeria. Spinal Cord 2009; 47: 134-137.

6 Hoque MF, Grangeon C, Reed K. Spinal cord lesions in Bangladesh: an epidemiological study 1994-1995. Spinal Cord 1999; 37: 858-861.

7 Ahidjo KA, Olayinka SA, Ayokunle O, Mustapha AF, Sulaiman GA, Gbolahan AT. Prehospital transport of patients with spinal cord injury in Nigeria. J Spinal Cord Med 2011; 34: 308-311.

8 Gureja Y, Opiyo W, Campbell E. Experiences with traumatic quadripleiga and paraplegia at Princess Marina Hospital Gaborone. J Med Dent Assoc Botswana 1993; 23: 19-20.

9 Idowu OK, Yinusa W, Gbadegesin SA, Adebule GT. Risk factors for pressure ulceration in a resource constrained spinal injury service. Spinal Cord 2011; 49: 643-647.

10 Eastwood EA, Hagglund KJ, Ragnarsson KT, Gordon WA, Marino RJ. Medical rehabilitation length of stay and outcomes for persons with traumatic spinal cord injury 1990-1997. Arch Phys Med Rehabil 1999; 80: 1457-1463.

11 Chen D, Apple DF, Hudson LM, Bode R. Medical complications during acute rehabiltition following spinal cord injury-current experience of the Model Systems. Arch Phys Med Rehabil 1999; 80: 1397-1401.

12 Finnerup NB, Norrbrink C, Trok K, Piehl F, Johannesen IL, Sørensen JC et al. Phenotypes and predictors of pain following traumatic spinal cord injury: a prospective study. J Pain 2014; 15: 40-48.

13 WHO. International Classification of Impairments, Disabilities, and Handicaps. WHO: Geneva, Switzerland, 1980, pp 182.

$14 \mathrm{Di}$ Benedetto P. Clean intermittent self-catheterization in neuro-urology. Eur J Phys Rehabil Med 2011; 47: 651-659.

15 Löfvenmark I, Norrbrink C, Nilsson-Wikmar L, Hultling C, Chakandinakira S, Hasselberg M. Traumatic spinal cord injury in Botswana: characteristics, aetiology and mortality. Spinal Cord 2015; 53: 150-154.

16 Waring WP, Biering-Sorensen F, Burns S, Donovan W, Graves D, Jha et al. 2009 Review and revisions of the International Standards for the Neurological Classification of Spinal Cord Injury. J Spinal Cord Med 2010; 33: 346-352.

17 International Spinal Cord Society. Available from www.iscos.org.uk/international-scidata-sets. Accessed 22 November 2015.

18 DeVivo MJ, Biering-Sørensen F, New P, Chen Y. Standardization of data analysis and reporting of results from the International Spinal Cord Injury Core Data Set. Spinal Cord 2011; 49: 596-599.

19 Divanoglou A, Westgren N, Bjelak S, Levi R. Medical conditions and outcomes at 1 year after acute traumatic spinal cord injury in a Greek and a Swedish region: a prospective, population-based study. Spinal Cord 2010; 48: 470-476.

20 Zakrasek EC, Creasey G, Crew JD. Pressure ulcers in people with spinal cord injury in developing nations. Spinal Cord 2015; 53: 7-13.

21 Fromovich-Amit Y, Biering-Sørensen F, Baskov V, Juocevicius A, Hansen HV, Gelernter I et al. Properties and outcomes of spinal rehabilitation units in four countries. Spinal Cord 2009; 47: 597-603.

22 Scheel-Sailer A, Wyss A, Boldt C, Post MW, Lay V. Prevalence, location, grade of pressure ulcers and association with specific patient characteristics in adult spinal cord injury patients during the hospital stay: a prospective cohort study. Spinal Cord 2013; 51: 828-833. 